\title{
Importance of residual primary cancer after induction therapy for esophageal adenocarcinoma
}

\author{
Siva Raja, MD, PhD, ${ }^{a}$ Thomas W. Rice, MD, ${ }^{a}$ John Ehrlinger, PhD, ${ }^{\mathrm{b}}$ John R. Goldblum, MD, \\ Lisa A. Rybicki, MS, ${ }^{\mathrm{b}}$ Sudish C. Murthy, MD, PhD, ${ }^{\mathrm{a}}$ David Adelstein, MD, ${ }^{\mathrm{d}}$ Gregory Videtic, MD, \\ Michael P. McNamara, MD, ${ }^{d}$ and Eugene H. Blackstone, $\mathrm{MD}^{\mathrm{a}, \mathrm{b}}$
}

\begin{abstract}
Objectives: To (1) assess the continuous distribution of the percentage of residual primary cancer in resection specimens after induction therapy for locally advanced esophageal adenocarcinoma, (2) determine the effects of residual primary cancer on survival after esophagectomy, (3) ascertain interplay between residual primary cancer and classical classifications of response to induction therapy (ypTNM), and (4) identify predictors of residual primary cancer.

Methods: From January 2006 to November 2012, 188 patients (78\%) underwent accelerated chemoradiotherapy, and 52 patients (22\%) underwent chemotherapy alone followed by esophagectomy for adenocarcinoma. Mean age was $61 \pm 9.2$ years, and $89 \%$ were male. Residual primary cancer, assessed as the percentage of residual primary cancer cells in resection specimens, was quantified histologically by a gastrointestinal pathologist. Random Forest technology was used for data analysis.
\end{abstract}

Results: Twenty-five specimens (10\%) had no residual primary cancer (ypT0), $79(33 \%)$ had $1 \%$ to $25 \%$ residual cancer, 91 (38\%) had $26 \%$ to $75 \%$, and 45 $(19 \%)$ had $>75 \%$. Survival was worse with increasing residual primary cancer, plateauing at $75 \%$. Greater residual primary cancer was associated with worse survival across the spectrum of higher ypTN. Higher ypT, larger number of positive nodes, and use of induction chemotherapy rather than induction chemoradiotherapy were associated with greater residual primary cancer.

Conclusions: Less residual primary cancer in response to preoperative therapy is associated with a linear increase in survival after esophagectomy for locally advanced esophageal adenocarcinoma; however, survival is poorer than for resected early-stage cancers. Therefore, for patients with poor prognostic indicators, including higher percentage of residual primary cancer, the role of adjuvant therapy needs to be further examined in an attempt to improve survival. (J Thorac Cardiovasc Surg 2016;152:756-61)

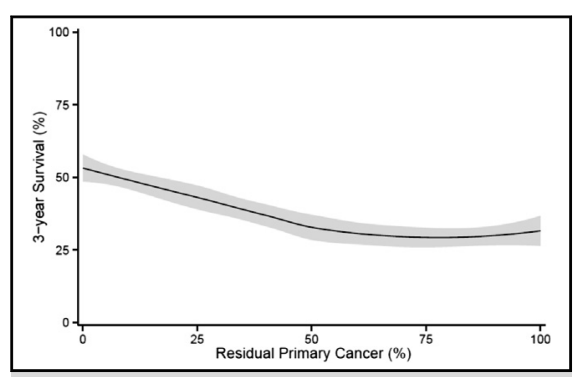

Predicted 3-year survival according to percentage of residual primary esophageal adenocarcinoma.

\section{Central Message}

Increasing amount of residual primary cancer after induction therapy is associated with worsening survival in esophageal adenocarcinoma.

\section{Perspective}

In the 7th edition of the American Joint Committee on Cancer Staging Manual, pathologic staging for adenocarcinoma of the esophagus inaccurately predicts survival for responders to induction therapy. This study describes the utility of residual primary cancer as a prognostic indicator for these patients.

See Editorial Commentary page 761.
Patients with esophageal adenocarcinoma responding to induction therapy (cancer downstaged) have better survival than nonresponders, ${ }^{1,2}$ and those with $<50 \%$ residual primary cancer in resection specimens are reported to have better survival than those with $>50 \%{ }^{3}$ However, percentage of residual primary cancer is a continuous variable.

From the ${ }^{\mathrm{a}}$ Department of Thoracic and Cardiovascular Surgery, Heart and Vascular Institute, ${ }^{b}$ Department of Quantitative Health Sciences, Research Institute, ${ }^{c}$ Department of Pathology, Pathology and Laboratory Medicine Institute, and Departments of ${ }^{\mathrm{d}}$ Hematology and Medical Oncology and ${ }^{\mathrm{e}}$ Radiation Oncology, Taussig Cancer Institute, Cleveland Clinic, Cleveland, Ohio.

This study was supported in part by the Gus P. Karos Registry Fund, the Kenneth Gee and Paula Shaw, PhD, Chair in Heart Research, held by Dr Blackstone, and the Daniel and Karen Lee Endowed Chair in Thoracic Surgery, held by Dr Murthy. Read at the 94th Annual Meeting of The American Association for Thoracic Surgery, Toronto, Ontario, Canada, April 26-30, 2014.
Thus, the objectives of this study were to (1) assess the continuous distribution of the percentage of residual primary cancer in resection specimens after induction therapy for locally advanced esophageal adenocarcinoma, (2) determine the effects of residual primary cancer on survival after esophagectomy, (3) ascertain the interplay between residual

Received for publication April 18, 2014; revisions received March 29, 2016; accepted for publication May 1, 2016

Address for reprints: Siva Raja, MD, PhD, Cleveland Clinic, 9500 Euclid Ave/Desk J4-1, Cleveland, OH 44195 (E-mail: rajas@ ccf.org). $0022-5223 / \$ 36.00$

Copyright (C) 2016 Published by Elsevier Inc. on behalf of The American Association for Thoracic Surgery

http://dx.doi.org/10.1016/j.jtcvs.2016.05.007 


\section{Abbreviation and Acronym}

VIMP $=$ variable importance

\section{Scanning this QR code will take you to appendices, supplemental figures, and tables for this article.}

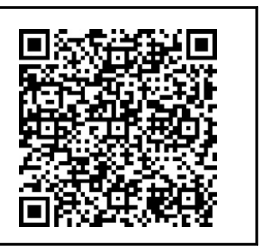

primary cancer and classical classifications of response to induction therapy (ypTNM), and (4) identify predictors of residual primary cancer.

\section{PATIENTS AND METHODS Study Population}

From January 2006 (when local recording of residual primary cancer was histologically standardized) to November 2012, 240 patients underwent induction therapy followed by esophagectomy for adenocarcinoma; $188(78 \%)$ received accelerated chemoradiotherapy and $52(22 \%)$ chemotherapy alone (Tables 1, 2, and E1). Of note, 17 patients had ypT4 or ypM1 disease. Patients with ypT4a disease had invasion of the diaphragm or direct invasion of the stomach distal to the primary cancer; those with ypT4b had invasion of either the airway or aorta. Patients with M1 disease had peritoneal nodules identified during resection at a point when the procedure could not be aborted; cM1 disease had not been identified. The Cleveland Clinic Institutional Review Board approved use of these data for research, with patient consent waived.

\section{Preoperative and Postoperative Therapy}

One hundred and eighty-eight patients received accelerated preoperative (induction) chemoradiotherapy, consisting of 4 days of cisplatin $\left(20 \mathrm{mg} \cdot \mathrm{m}^{-2} \cdot \mathrm{d}^{-1}\right)$ and fluorouracil $\left(1000 \mathrm{mg} \cdot \mathrm{m}^{-2} \cdot \mathrm{d}^{-1}\right)$, and concurrent hyperfractionated radiation therapy ( 1.5 Gy twice daily to a total dose of $30 \mathrm{~Gy}$ ). ${ }^{4}$ Of these, 127 received a similar course of postoperative (adjuvant) chemoradiotherapy; 1 patient received adjuvant chemotherapy alone, 2 adjuvant radiotherapy alone, and 58 no adjuvant therapy.

Fifty-two patients received 3 courses of induction chemotherapy, consisting of epirubicin $\left(50 \mathrm{mg} \cdot \mathrm{m}^{-2}\right)$ and oxaliplatin $\left(130 \mathrm{mg} \cdot \mathrm{m}^{-2}\right)$ on day 1 and fluorouracil $\left(200 \mathrm{mg} \cdot \mathrm{m}^{-2} \cdot \mathrm{d}^{-1}\right)$ as a continuous intravenous infusion for 21 days. $^{5}$ Cycles were repeated every 3 weeks. Of these, 39 received adjuvant chemoradiotherapy, consisting of 2 cycles of cisplatin $\left(80 \mathrm{mg} \cdot \mathrm{m}^{-2}\right)$ and fluorouracil $\left(4000 \mathrm{mg} \cdot \mathrm{m}^{-2}\right)$, and concurrent radiation therapy to a total dose of $50-55 \mathrm{~Gy} ; 1$ patient received adjuvant chemotherapy alone and 12 no adjuvant therapy.

\section{Follow-up}

Median follow-up was 1.1 years. Median follow-up for surviving patients was 1.7 years, with $25 \%$ followed more than 3.0 years and $10 \%$ more than 4.8 years. Median potential follow-up, if there were no deaths, was 2.3 years $(25 \%$ more than 3.5 years and $10 \%$ more than 5.8 years $){ }^{6}$

\section{Pathologic Analysis}

Residual primary cancer was measured by percentage of residual primary cancer cells in the resection specimen. Esophagectomy specimens were examined by pathologists specializing in gastrointestinal pathology. Specimens were evaluated using light microscopy after hematoxylin and
TABLE 1. Patient characteristics (total $n=240$ )

\begin{tabular}{lcc}
\hline \multicolumn{1}{c}{ Variable } & $\mathbf{n}^{*}$ & Number $(\%)$ or mean \pm SD \\
\hline Demographics & & \\
Age $(\mathrm{y})$ & 240 & $61 \pm 9.0$ \\
Male & 240 & $214(89)$ \\
White & 240 & $229(95)$ \\
$\quad$ Body mass index $\left(\mathrm{kg} \cdot \mathrm{m}^{-2}\right)$ & 195 & $28 \pm 5.6$ \\
Comorbidities & & \\
$\quad$ Diabetes & 196 & $35(18)$ \\
Coronary artery disease & 232 & $34(15)$ \\
Hypertension & 196 & $96(49)$ \\
Peripheral arterial disease & 232 & $6(2.6)$ \\
Smoking history & 176 & $135(73)$ \\
FEV $(\%$ of predicted) & 236 & $93 \pm 16$ \\
FVC $(\%$ of predicted $)$ & 236 & $98 \pm 14$ \\
Creatinine $\left(\mu\right.$ mol $\left.\cdot \mathrm{L}^{-1}\right)$ & 142 & $79 \pm 22$ \\
Bilirubin $\left(\mu\right.$ mol $\left.\cdot \mathrm{L}^{-1}\right)$ & 188 & $5.1 / 6.8 / 12 \dagger$ \\
\hline
\end{tabular}

$S D$, Standard deviation; $F E V_{l}$, forced expiratory volume in 1 second; $F V C$, forced vital capacity. *Patients with data available. $\dagger 15$ th/50th/85th percentiles.

eosin staining. A minimum of 4 blocks of the primary cancer were examined, but this number increased with increasing cancer size. Percentage of residual primary cancer cells was scored in accordance with previously published histologic criteria that were standardized by a gastrointestinal pathologist (J.R.G.), ${ }^{7}$ but using $1 \%$ increments for values $0 \%$ to $5 \%$ and then $5 \%$ increments for the remainder. Regional lymph nodes were not scored for residual primary cancer.

\section{Data Analysis}

Random Forest analysis. We used Random Forest technology as the analytic strategy in part to avoid restrictive parametric modeling assumptions, given no prior knowledge of what relationships might exist, and in part because we previously demonstrated with this method that there is a complex interplay between esophageal cancer characteristics and survival. ${ }^{8}$ A Random Forest is a collection of decision-tree analyses, wherein a variable is chosen to optimally split the population to improve prediction. This process is applied recursively to create a tree (recursive partitioning, ${ }^{9}$ classification and regression trees ${ }^{10}$ ). Individual trees "grown" by this method are inherently unstable, but this can be mitigated by creating a collection of trees from bootstrap samples of the original dataset (the bootstrap dataset is formed by random sampling of patients with replacement until a dataset of equal size is generated; some patients will be duplicated, and an average of $37 \%$ will not be sampled). Subsequently, an ensemble average can be formulated across this forest of individual trees. The validity of the forest is evaluated by assessing outcomes of patients who were not selected in the bootstrap process, resulting in internal multifold cross-validation. This transforms variables associated with an outcome of interest into predictors of that outcome.

Because values were missing for some variables, Random Forest imputation was used to maximize use of available data. ${ }^{11}$

Variable selection. Rather than $P$ values, 2 metrics of prediction accuracy are generated. The first ranks the importance of each variable in predicting the outcome of interest (variable importance, or VIMP) based on the patients not selected (called the "out-of-bag" or holdout samples). ${ }^{12}$ The second quantifies the average number of branches before a variable is split (called "minimal depth"): The closer to the trunk of the tree a variable is split, the more important that variable is to prediction accuracy. ${ }^{13}$

In summary, predictors of outcome using Random Forest technology are identified in 2 steps: (1) building the forest based on residual primary cancer and other patient characteristics and the outcome of interest, and (2) using the resulting forest to discover the importance of variables to 
TABLE 2. Cancer classification and treatment (total $\mathbf{n}=240$ )*

\begin{tabular}{|c|c|}
\hline Variable & Number $(\%)$ \\
\hline \multicolumn{2}{|l|}{ Cancer characteristics } \\
\hline Location: lower esophagus/GEJ & $239(99.6)$ \\
\hline \multicolumn{2}{|l|}{ Preinduction therapy clinical stage $\dagger$} \\
\hline \multicolumn{2}{|l|}{ EUS cT classification } \\
\hline cTis & $3(1.3)$ \\
\hline cT1 & $8(3.4)$ \\
\hline cT2 & $62(26)$ \\
\hline cT3 & $158(67)$ \\
\hline cT4 & $4(1.7)$ \\
\hline $\mathrm{cN}+$ & $106(45)$ \\
\hline cM1 & $0(0)$ \\
\hline \multicolumn{2}{|l|}{ Induction therapy } \\
\hline Chemotherapy alone & $52(22)$ \\
\hline Chemoradiotherapy & $188(78)$ \\
\hline \multicolumn{2}{|l|}{ Esophagectomy } \\
\hline Left thoracoabdominal & $230(96)$ \\
\hline Transhiatal & $10(4.0)$ \\
\hline R0 resection & $212(88)$ \\
\hline $\mathrm{R} 1$ resection & $28(12)$ \\
\hline Radial only & $15(54)$ \\
\hline Proximal or distal only & $8(29)$ \\
\hline Both & $5(18)$ \\
\hline Number of lymph nodes resected & $27 \pm 13 \ddagger$ \\
\hline \multicolumn{2}{|l|}{ Pathologic TNM } \\
\hline \multicolumn{2}{|l|}{ урТ } \\
\hline 0 & $25(10)$ \\
\hline 1 & $13(5.4)$ \\
\hline 2 & $33(14)$ \\
\hline 3 & $158(66)$ \\
\hline $4 a$ & $8(3.3)$ \\
\hline $4 \mathrm{~b}$ & $3(1.2)$ \\
\hline \multicolumn{2}{|l|}{ ypN } \\
\hline 0 & $88(37)$ \\
\hline 1 & $57(24)$ \\
\hline 2 & $53(22)$ \\
\hline 3 & $42(18)$ \\
\hline Extracapsular lymph node invasion & $34 / 166(20)$ \\
\hline \multicolumn{2}{|l|}{ ypM } \\
\hline 0 & 237 (99) \\
\hline 1 & $3(1.2)$ \\
\hline Lymphovascular invasion & $111 / 231(48)$ \\
\hline \multicolumn{2}{|l|}{ Stage groupings } \\
\hline 0 & $19(7.9)$ \\
\hline IA & $4(1.7)$ \\
\hline IB & $10(4.2)$ \\
\hline IIA & $9(3.8)$ \\
\hline IIB & $60(25)$ \\
\hline IIIA & $46(19)$ \\
\hline IIIB & $41(17)$ \\
\hline IIIC & $45(19)$ \\
\hline IV & $6(2.5)$ \\
\hline
\end{tabular}

GEJ, Gastroesophageal junction; $E U S$, endoscopic ultrasonography; $T N M$, tumor-nodemetastasis. *Classified according to the American Joint Committee on Cancer Staging Manual, 7 th edition. $\dagger$ Available for 235 patients. $\ddagger$ Mean \pm standard deviation. the prediction of outcome and their interrelationships with respect to outcome (Appendix E1).

Residual primary cancer and survival. A random survival forest was constructed with 2000 trees. At each node, all variables were used (Table E2), but 4 candidate variables were randomly selected from these for splitting each node (which avoids the statistical problem related to the order in which variables are incorporated into models).

Interplay of residual primary cancer, ypTNM, and survival. We used variable dependence and partial (risk-adjusted) dependence to determine how variables were related to patient survival. ${ }^{14}$ Conditional dependence and conditional partial dependence plots were used to detect interactions between ypTNM and residual primary cancer.

Predictors of residual primary cancer. A Random Forest for regression was used to determine which variables were important for predicting the percentage of residual primary cancer.

\section{RESULTS}

\section{Residual Primary Cancer After Induction Therapy}

Twenty-five patients $(10 \%)$ had no residual primary cancer (ypT0), $79(33 \%)$ had $1 \%$ to $25 \%, 91(38 \%)$ had $26 \%$ to $75 \%$, and $45(19 \%$ ) had $>75 \%$ (see Figure E1 for detailed distribution).

\section{Residual Primary Cancer and Survival}

Survival after esophagectomy following induction therapy was $97 \%$ at 30 days, $92 \%$ at 90 days, and $68 \%$, $37 \%$, and $29 \%$ at 1,3 , and 5 years, respectively (Figure E2). It was worse with increasing residual primary cancer (Figures 1 and 2). This effect plateaued after approximately $75 \%$ residual primary cancer. Residual primary cancer was the 5th most important predictor of survival after esophagectomy and 3rd among cancer characteristics, the most important being number of positive nodes, followed by higher ypT (Figure E3). Total number of positive nodes was more important than residual primary cancer by a factor of 12 .

\section{Interplay of Residual Primary Cancer, ypTNM, and Survival}

Increasing percentage of residual primary cancer was associated with worse survival in all ypT classifications at 6 months, 1 year, and 3 years (Figure 3). Survival was better with earlier ypT classifications (ypT0-2) than more advanced ypT (ypT3-4). Increasing percentage of residual primary cancer was also associated with worse survival in all ypN classifications at 6 months, 1 year, and 3 years (Figure 4); however, with increasing ypN classification, the effect was blunted.

\section{Predictors of Residual Primary Cancer}

The most important predictor of residual primary cancer after induction therapy was increasing ypT classification, followed by increasing number of positive nodes, 


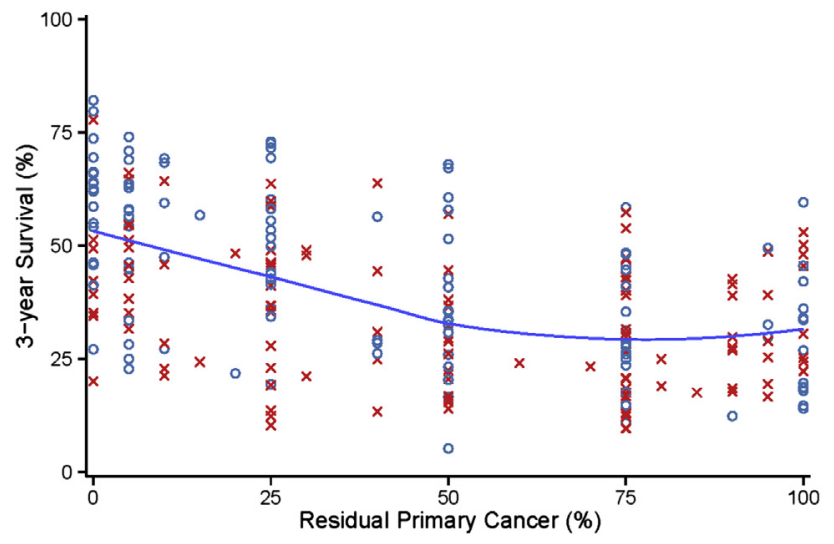

FIGURE 1. Predicted 3-year survival according to the percentage of residual primary cancer adjusted for all patient, cancer, and treatment characteristics. Red x's represent deaths and blue circles represent living or censored patients.

lymphovascular invasion, induction chemotherapy alone, and higher histologic grade (Figure E4). Preoperative chemoradiotherapy was associated with $10 \%$ less residual primary cancer than preoperative chemotherapy alone (Figure 5). Regardless of modality of induction therapy, there was a near linear increase in residual primary cancer up to 10 lymph nodes, after which the relationship plateaued.

\section{DISCUSSION}

\section{Principal Findings}

Survival after esophagectomy following induction therapy is worse with increasing residual primary cancer for all ypT and ypN classifications; however, its effect is greater for earlier ypT and ypN. The most important predictor of residual primary cancer after induction therapy was ypT, followed by lymph node invasion. Adding radiotherapy to induction chemotherapy resulted in less residual primary cancer.

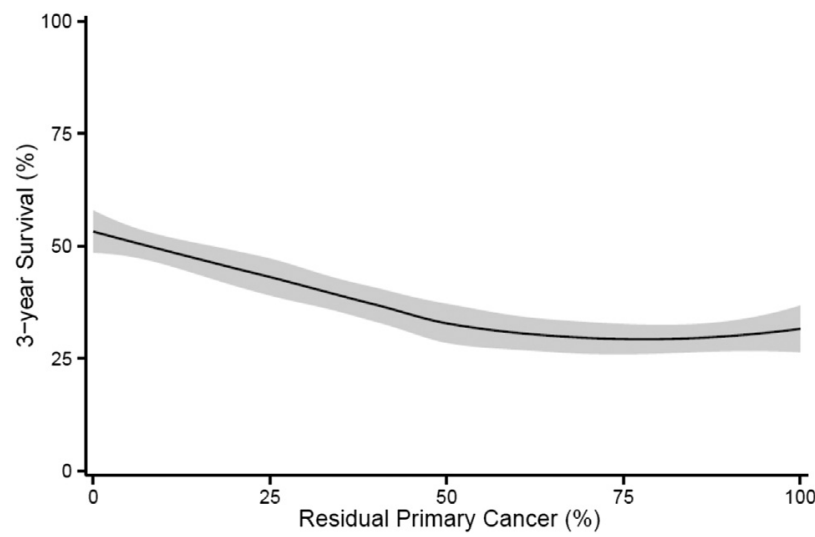

FIGURE 2. Predicted 3-year survival according to percentage of residual primary esophageal adenocarcinoma.

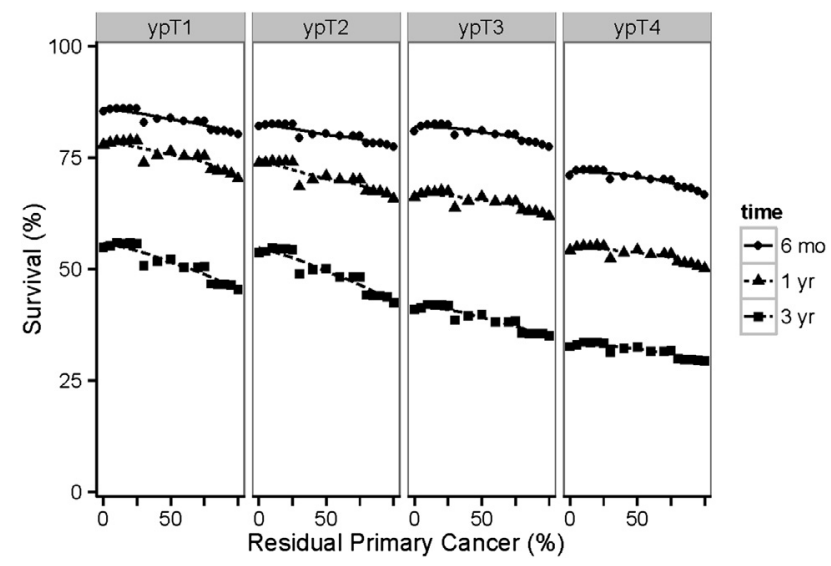

FIGURE 3. Partial dependence of 6-month, 1-year, and 3-year survival on residual primary cancer stratified by ypT classifications. Symbols are point estimates at 6 months (dots), 1 year (triangles), and 3 years (squares).

\section{Findings in Context}

Complete pathologic response after induction therapy is associated with improved patient survival, although not to levels of patients without cancer or even early-stage esophageal cancer. ${ }^{1,2,16,17}$ Complete primary cancer response has been reported to range from $22 \%$ to $33 \%,{ }^{18-20}$ whereas in our study it was only $10 \%$. The difference may be attributable to our unconventional, accelerated chemoradiation protocol, which permits surgery 6 to 8 weeks after commencing induction therapy. ${ }^{4,5}$ This is in contrast to conventional induction protocols of 2 to 3 courses of induction chemotherapy and concurrent radiation that delay surgery for 12 to 18 weeks after induction. Thus, early esophagectomy, before induction-induced cell death is complete, may account for the lower response observed in our study. Nevertheless, complete responders had better

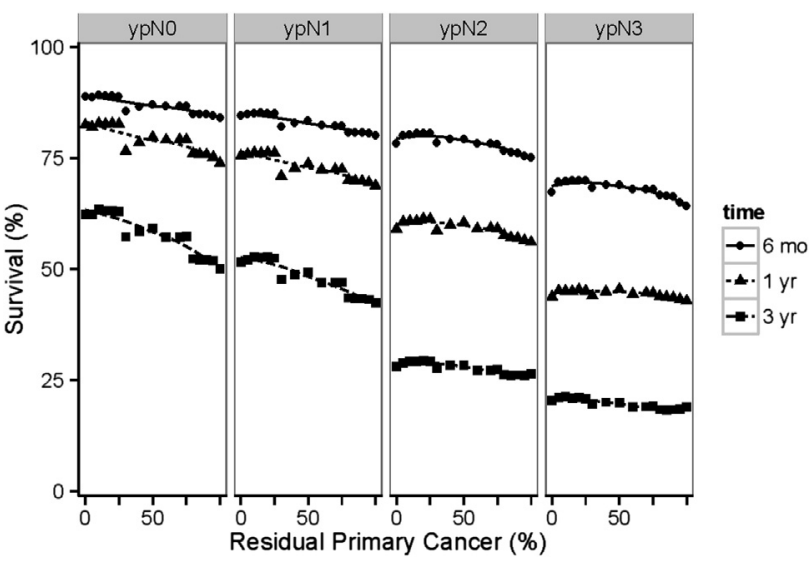

FIGURE 4. Partial dependence of 6-month, 1-year, and 3-year survival on residual primary cancer stratified by ypN classifications. Symbols are point estimates at 6 months (dots), 1 year (triangles), and 3 years (squares), with loess curves indicating trends. Note: ypN1 is 1 to 2 positive nodes, ypN2 is 3 to 6 positive nodes, and ypN3 is 7 or more positive nodes. ${ }^{15}$ 


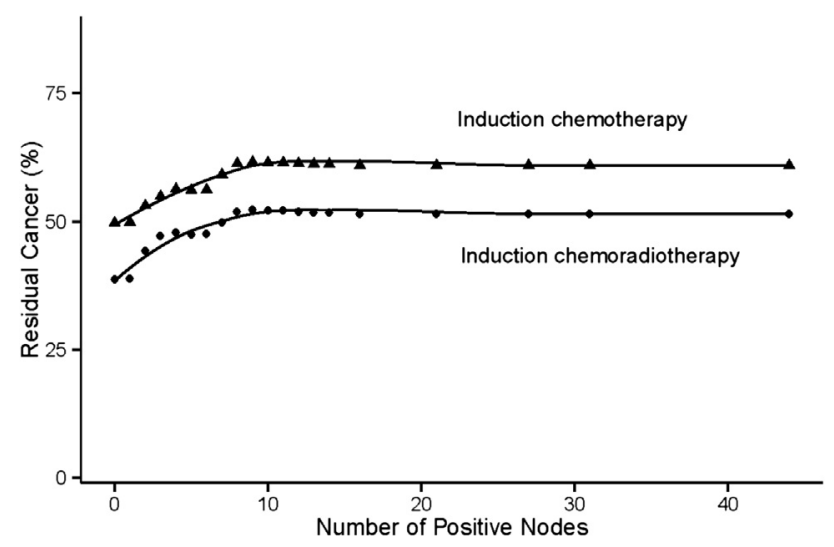

FIGURE 5. Relationship of the number of positive lymph nodes and residual primary cancer according to modality of induction therapy.

survival than those who had residual primary cancer. Therefore, it is possible that we have identified a group of patients whose cancer is highly sensitive to induction therapy, resulting in complete response to abbreviated therapy and an accelerated time course.

Partial response to treatment has also been associated with improved survival. ${ }^{1,3}$ We show a linear decrease in survival with increasing residual primary cancer until it exceeds $75 \%$. In addition, we demonstrate that although residual primary cancer is a predictor of survival, the magnitude of its effect is eclipsed by other ypT and ypN.

An alternative treatment strategy for patients with increased residual primary cancer is needed, because these patients are likely to have a worse outcome with current treatments. This may involve adjuvant therapy for those who have completed their full course of therapy preoperatively. Furthermore, different chemotherapeutic agents can be used for adjuvant therapy. Perhaps with improvements in molecular medicine, residual primary cancer can be tested for chemosensitivity to different regimens in an effort to improve outcomes. There is also a need for new diagnostic modalities that are more sensitive in assessing response to therapy than conventional modalities, such as positron emission tomography scans and endoscopy, which have poor sensitivity. ${ }^{21,22}$

In our study, adding radiotherapy to chemotherapy preoperatively resulted in decreased residual primary cancer.

\section{Strengths and Limitations}

This is a single-institution study reflecting outcomes of a highly selected patient population undergoing a standardized regimen of induction therapy. Therefore, other induction regimens using different treatment schedules and agents can potentially generate more complete responders. These data are derived from a prospectively collected database that was analyzed retrospectively using a modern machine-learning method, Random Forests, capable of accounting for complex interactions without making model assumptions and providing extensive internal validation of the results. Although there are a reasonable number of patients in this study, a larger cohort may be necessary to identify other associations. Nevertheless, our results add to the understanding of the role that residual primary cancer plays in patient survival while accounting for other patient or cancer characteristics in esophageal adenocarcinoma.

\section{Conclusions and Recommendations}

Decreased residual primary cancer in response to preoperative therapy is associated with a linear increase in survival after esophagectomy for locally advanced esophageal adenocarcinoma; however, survival is poorer than for resected early-stage cancers. Therefore, for patients with poor prognostic indicators, including a higher percentage of residual primary cancer, the role of adjuvant therapy needs to be further examined in an attempt to improve their poor survival. The percentage of residual primary cancer should also be considered as a possible prognostic variable in refining esophageal cancer staging. Reliance on induction therapy alone seems to be inadequate, and additional adjuvant strategies should be considered.

\section{Conflicts of Interest Statement}

Authors have nothing to disclose with regard to commercial support.

The authors thank Jiayan He, ScD, for statistical expertise and support.

\section{References}

1. Scheer RV, Fakiris AJ, Johnstone PA. Quantifying the benefit of a pathologic complete response after neoadjuvant chemoradiotherapy in the treatment of esophageal cancer. Int J Radiat Oncol Biol Phys. 2011;80:996-1001.

2. Rice TW, Blackstone EH, Adelstein DJ, Zuccaro G Jr, Vargo JJ, Goldblum JR, et al. N1 esophageal carcinoma: the importance of staging and downstaging. J Thorac Cardiovasc Surg. 2001;121:454-64.

3. Francis AM, Sepesi B, Correa AM, Blum MA, Erasmus JJ, Lee JH, et al. The influence of histopathologic tumor viability on long-term survival and recurrence rates following neoadjuvant therapy for esophageal adenocarcinoma. Ann Surg. 2013;258:500-7.

4. Adelstein DJ, Rice TW, Rybicki LA, Saxton JP, Videtic GM, Murthy SC, et al. A phase II trial of accelerated multimodality therapy for locoregionally advanced cancer of the esophagus and gastroesophageal junction: the impact of clinical heterogeneity. Am J Clin Oncol. 2007;30:172-80.

5. McNamara MJ, Adelstein DJ, Bodmann JW, Greskovich JF Jr, Ives DI, Mason DP, et al. A phase II trial of induction epirubicin, oxaliplatin, and fluorouracil, followed by surgery and postoperative concurrent cisplatin and fluorouracil chemoradiotherapy in patients with locoregionally advanced adenocarcinoma of the esophagus and gastroesophageal junction. J Thorac Oncol. 2014;9:1561-7.

6. Goldman AI. Eventcharts: visualizing survival and other timed-event data. Am Statistician. 1992;46:13-8.

7. Chang F, Deere H, Mahadeva U, George S. Histopathologic examination and reporting of esophageal carcinomas following preoperative neoadjuvant therapy: practical guidelines and current issues. Am J Clin Pathol. 2008;129: 252-62.

8. Ishwaran H, Blackstone EH, Apperson-Hansen C, Rice TW. A novel approach to cancer staging: application to esophageal cancer. Biostatistics. 2009;10:603-20. 
9. Zhang H, Singer B. Recursive Partitioning Analysis in the Health Sciences. New York, NY: Springer-Verlag; 1999.

10. Breiman L, Friedman JH, Olshen R, Stone C. Classification and Regression Trees. Monterey, CA: Wadsworth and Brooks; 1984.

11. Ishwaran H, Kogalur UB, Blackstone EH, Lauer MS. Random survival forests. Ann Appl Stat. 2008:2:841-60.

12. Breiman L. Random forests. Machine Learn. 2001;45:5-32.

13. Ishwaran H, Kogalur UB, Gorodeski EZ, Minn AJ, Lauer MS. High-dimensional variable selection for survival data. J Am Stat Assoc. 2010;105:205-17.

14. Ehrlinger J. "ggRandomForests": visually exploring Random Forests. R package version 1.1.4. http://cran.r-project.org/package $=$ ggRandomForests. Accessed December 12, 2015.

15. Edge SB, Byrd DR, Compton CC, Fritz AG, Greene FL, Trotti A, eds. American Joint Committee on Cancer Staging Manual. New York, NY: Springer-Verlag; 2010.

16. Ajani JA, Correa AM, Hofstetter WL, Rice DC, Blum MA, Suzuki A, et al. Clinical parameters model for predicting pathologic complete response following preoperative chemoradiation in patients with esophageal cancer. Ann Oncol. 2012;23:2638-42.

17. Swisher SG, Winter KA, Komaki RU, Ajani JA, Wu TT, Hofstetter WL, et al. A Phase II study of a paclitaxel-based chemoradiation regimen with selective surgical salvage for resectable locoregionally advanced esophageal cancer: initial reporting of RTOG 0246. Int J Radiat Oncol Biol Phys. 2012;82:1967-72.
18. Donington JS, Miller DL, Allen MS, Deschamps C, Nichols FC III, Pairolero PC Tumor response to induction chemoradiation: influence on survival after esophagectomy. Eur J Cardiothorac Surg. 2003;24:631-6; discussion 6-7.

19. Rizzetto C, DeMeester SR, Hagen JA, Peyre CG, Lipham JC, DeMeester TR. En bloc esophagectomy reduces local recurrence and improves survival compared with transhiatal resection after neoadjuvant therapy for esophageal adenocarcinoma. J Thorac Cardiovasc Surg. 2008;135:1228-36.

20. Gu Y, Swisher SG, Ajani JA, Correa AM, Hofstetter WL, Liao Z, et al. The number of lymph nodes with metastasis predicts survival in patients with esophageal or esophagogastric junction adenocarcinoma who receive preoperative chemoradiation. Cancer. 2006;106:1017-25.

21. Stiles BM, Salzler G, Jorgensen A, Nasar A, Paul S, Lee PC, et al Complete metabolic response is not uniformly predictive of complete pathologic response after induction therapy for esophageal cancer. Ann Thorac Surg. 2013 96:1820-5.

22. Yang Q, Cleary KR, Yao JC, Swisher SG, Roth JA, Lynch PM, et al. Significance of post-chemoradiation biopsy in predicting residual esophageal carcinoma in the surgical specimen. Dis Esophagus. 2004;17:38-43.

Key Words: esophageal carcinoma, residual primary cancer, induction therapy, survival

\title{
EDITORIAL COMMENTARY
}

\section{For esophageal cancer, biology is still king}

\author{
Thomas Ng, MD, FRCSC, FACS
}

From the Department of Surgery, The Warren Alpert Medical School of Brown University, Providence, RI Disclosures: Author has nothing to disclose with regard to commercial support.

Received for publication May 22, 2016; accepted for publication May 26, 2016; available ahead of print June 18, 2016.

Address for reprints: Thomas Ng, MD, FRCSC, FACS, 2 Dudley St, Suite 470, Providence, RI 02905 (E-mail: tng@usasurg.org).

J Thorac Cardiovasc Surg 2016;152:761-2

$0022-5223 / \$ 36.00$

Copyright $\subset 2016$ by The American Association for Thoracic Surgery

http://dx.doi.org/10.1016/j.jtcvs.2016.05.045

It has long been known that pathologic response complete (pCR) to induction therapy represents the best scenario for long-term survival in patients with advanced esophageal cancer. In a randomized study published in 2001, Urba and colleagues $^{1}$ reported a pCR rate of $28 \%$, with a $64 \% 3$-year survival for those achieving pCR after induction chemoradiation. In this issue of the Journal, Raja and colleagues $^{2}$ go further with this concept, reporting a linear trend of worsening survival with increasing residual cancer after induction therapy. The authors also evaluated the predictors of residual cancer and the predictors of survival by using Random Forest technology, a nonparametric analysis that better identifies and handles complex interactions among variables in the study of esophageal cancer. This similar statistical analysis was used to develop

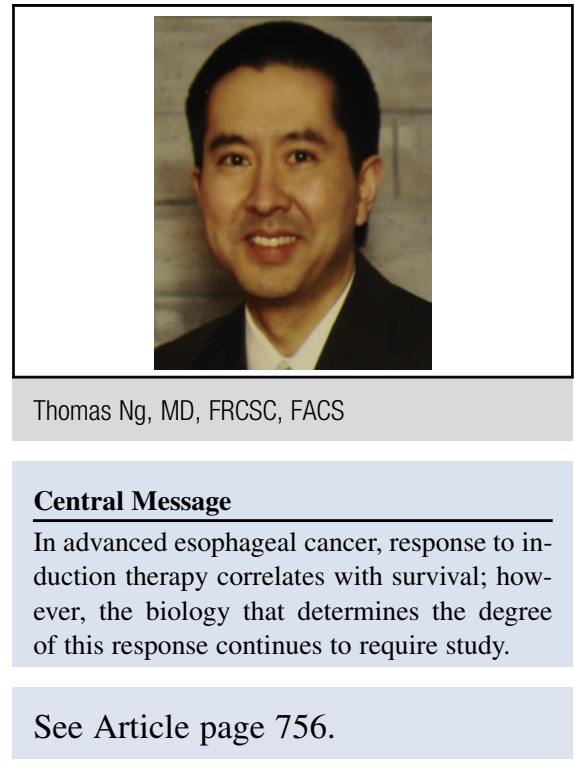

the American Joint Committee on Cancer seventh edition staging for esophageal cancer. ${ }^{3}$

Readers of this article may question the applicability of this study to their own practice of managing patients with advanced esophageal cancer. The 2 induction treatment 
APPENDIX E1. RANDOM FOREST TECHNOLOGY A Random Forest ${ }^{\mathrm{E} 1}$ is grown by bagging ${ }^{\mathrm{E} 2}$ a collection of classification and regression trees (CART). ${ }^{\mathrm{E} 3}$ The method uses a set of B bootstrap ${ }^{\mathrm{E} 4}$ samples from the original data, growing an independent CART model on each subsample of the population. Each tree is grown by recursively partitioning the population based on optimization of a split rule over the P-dimensional covariable space. At each split, a subset of $\mathrm{m} \leq \mathrm{P}$ candidate variables are tested for the split rule optimization, dividing each node population into 2 daughter node populations. Each daughter node is then split again until the process reaches the stopping criteria, node purity, or minimal node member size, which defines the set of terminal (unsplit) nodes for the tree. In regression trees, node impurity is measured by mean squared error, whereas in classification problems the Gini index is used. ${ }^{\mathrm{E} 5}$ Random Forest methodology sorts each observation into 1 unique terminal node within each tree, and estimates are constructed from the terminal node members. The Random Forest estimate for each observation is then calculated by aggregating, averaging (regression) or votes (classification), the terminal node results across the all B trees.

Random Survival Forests ${ }^{\mathrm{E} 6 \mathrm{E} 7}$ are an extension of Breiman's Random Forest for right-censored, time-to-event data. A forest of survival trees is grown using a log-rank splitting rule to select the optimal candidate variables. Survival estimates for each observation are constructed using a Kaplan-Meier estimator within each terminal node at each event time.

\section{E-References}

E1. Breiman L. Random Forests. Machine Learn. 2001;45:5-32.

E2. Breiman L. Bagging predictors. Machine Learn. 1996;24:123-40.

E3. Breiman L, Friedman JH, Olshen R, Stone C. Classification and Regression Trees. Monterey, CA: Wadsworth and Brooks; 1984.

E4. Efron B, Tibshirani RJ. An Introduction to the Bootstrap. New York, NY: Chapman and Hall/CRC; 1994.

E5. Friedman JH. Greedy function approximation: a gradient boosting machine. Ann Statist. 2000;29:1189-232.

E6. Ishwaran H, Kogalur UB, Gorodeski EZ, Minn AJ, Lauer MS. High-dimensional variable selection for survival data. J Am Stat Assoc. 2010;105:205-17.

E7. Ishwaran H, Kogalur UB. Random survival forests for R. Rnews. 2007;7:25-31. 


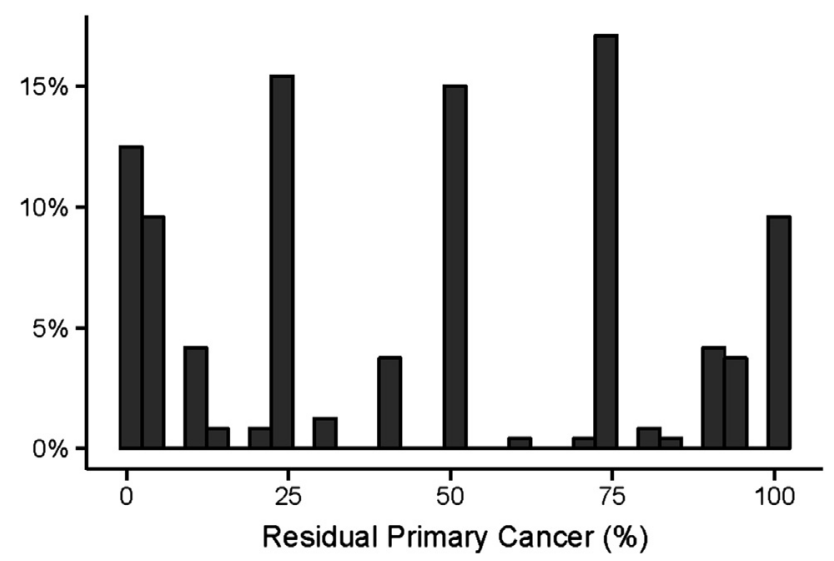

FIGURE E1. Distribution of residual primary cancer after induction therapy.

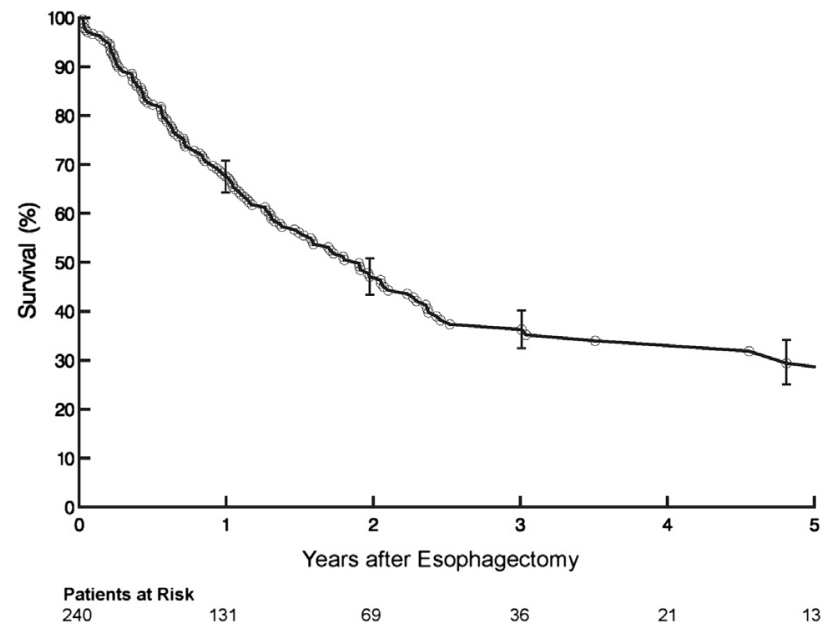

FIGURE E2. Risk-unadjusted survival after induction therapy and esophagectomy for adenocarcinoma of the esophagus. Each symbol represents a death, and vertical bars represent $68 \%$ confidence limits equivalent to \pm 1 standard error. 


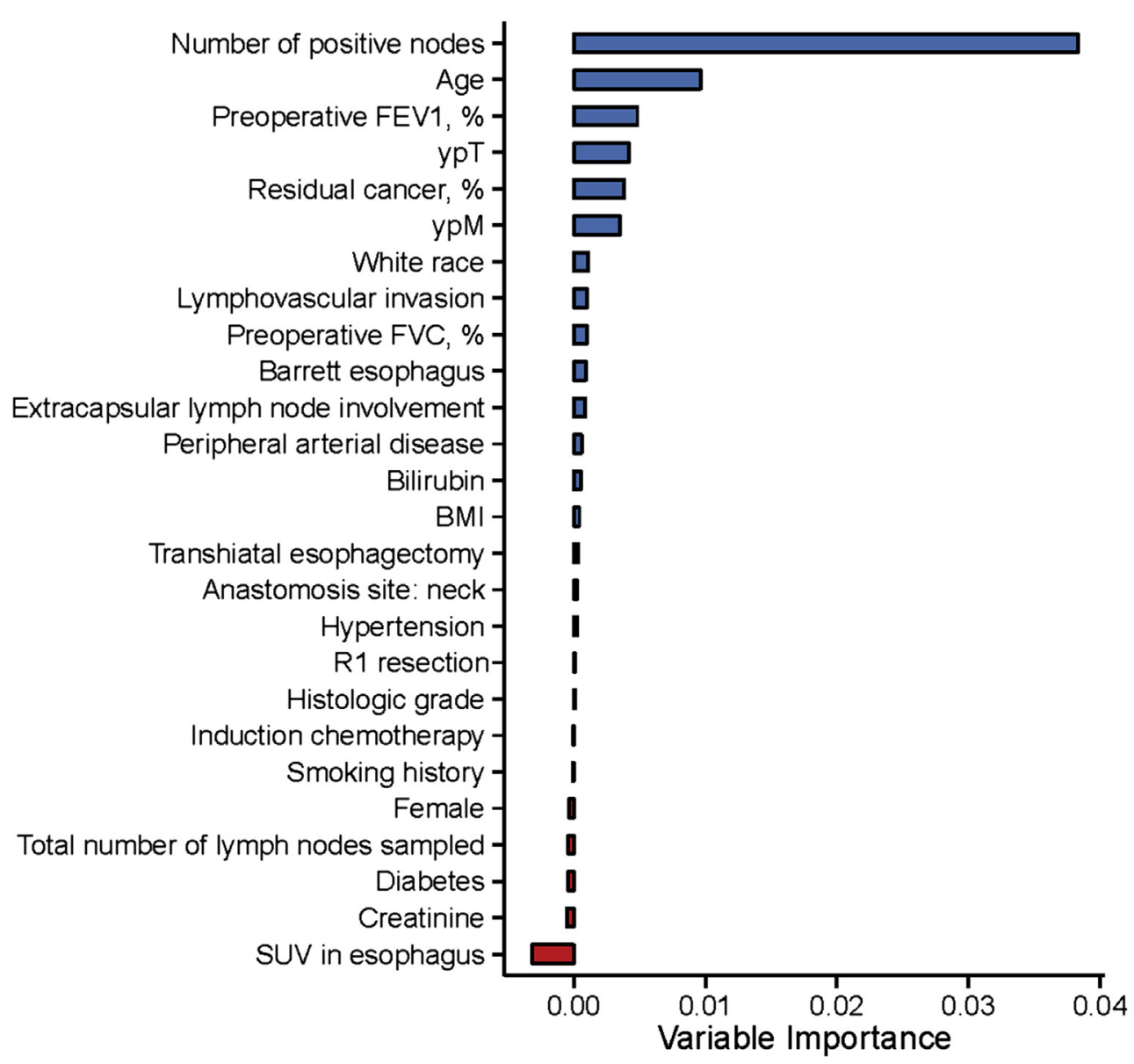

FIGURE E3. Variable importance of patient, cancer, and treatment characteristics for survival after esophagectomy. Blue bars represent a positive contribution to reducing prediction error, and red bars to the left of zero represent variables degrading prediction of survival. $F E V_{l}$, Forced expiratory volume in 1 second; $F V C$, forced vital capacity; $B M I$, body mass index; $S U V$, standardized uptake value. 


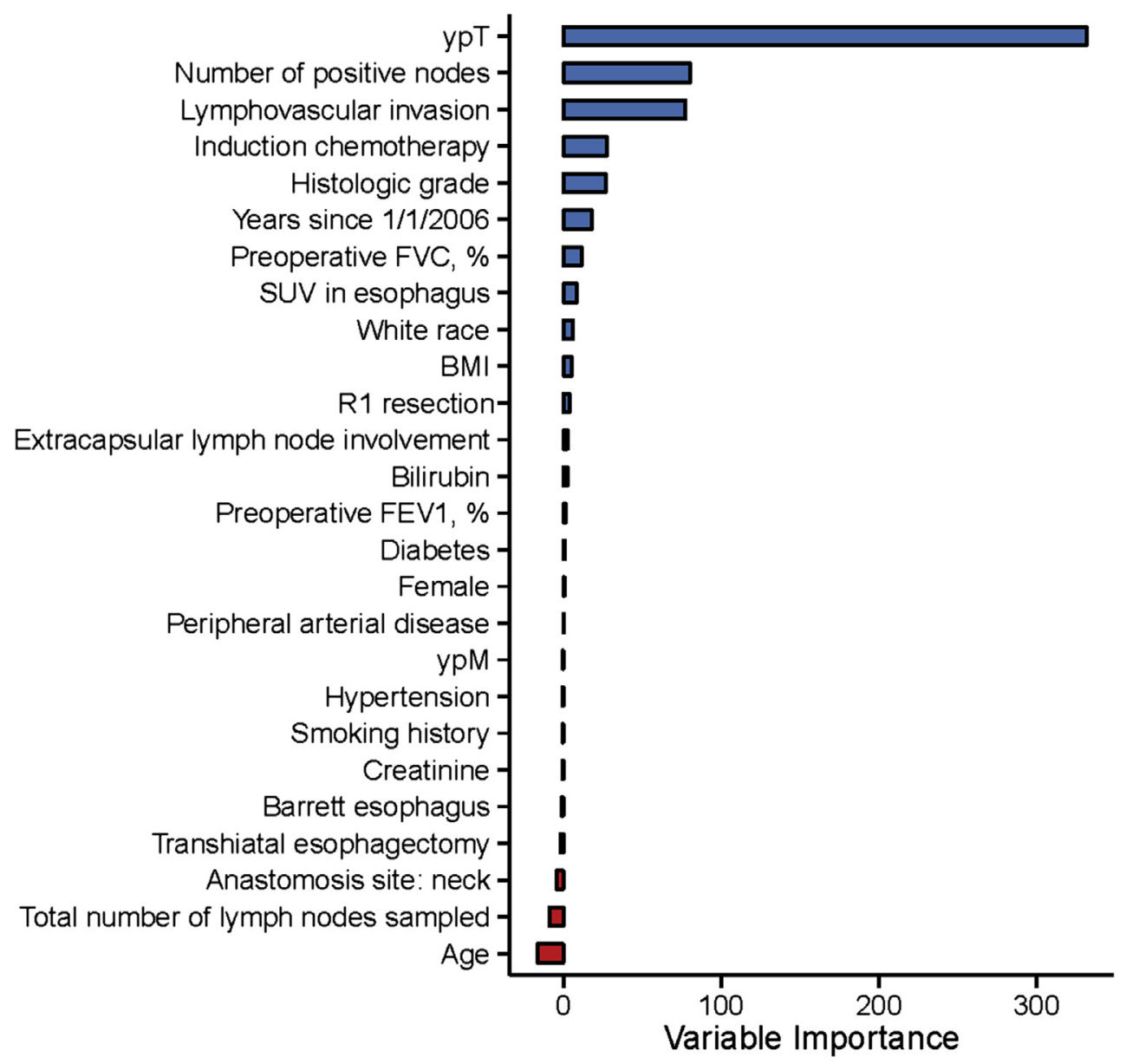

FIGURE E4. Variable importance of patient and cancer characteristics for residual primary cancer. Format is as in Figure E3. FVC, Forced vital capacity; $S U V$, standardized uptake value; $B M I$, body mass index; $F E V_{l}$, forced expiratory volume in 1 second.

TABLE E1. Postesophagectomy in-hospital events*

Event

Atrial fibrillation

Respiratory failure

Pleural effusion

Wound infection

Deep vein thrombosis

Pneumonia

Delirium

Anastomotic leak

Aspiration

Pneumothorax

Gastrointestinal bleeding

Pulmonary embolism

Vocal cord paralysis

Any complication

*Not mutually exclusive.
Number $(\%$ of 240$)$

42 (18)

28 (12)

26 (11)

$20(8.3)$

$19(7.9)$

$16(6.7)$

$14(5.8)$

$13(5.4)$

$6(2.5)$

$8(3.3)$

$4(1.7)$

$3(1.2)$

$1(0.42)$

$115(48)$

761.e4 The Journal of Thoracic and Cardiovascular Surgery • September 2016 
TABLE E2. Variables included in Random Forest analyses

Demographic

Comorbidities

Cancer characteristics

Therapy and surgical approach
Age $(\mathrm{y})$, sex, race, body mass index $\left(\mathrm{kg} \cdot \mathrm{m}^{-2}\right)$

History of diabetes, coronary artery disease, hypertension, peripheral arterial disease, smoking history, creatinine $\left(\mu \mathrm{mol} \cdot \mathrm{L}^{-1}\right)$, bilirubin $\left(\mu \mathrm{mol} \cdot \mathrm{L}^{-1}\right)$, forced expiratory volume in 1 second $(\%$ of predicted), forced vital capacity ( $\%$ of predicted)

Barrett esophagus, preoperative standardized uptake value in esophagus, site of anastomosis (neck, chest), pathologic T staging, pathologic M staging, histologic grade (G0, G1, G2, G3), extracapsular lymph node involvement, lymphovascular invasion, R1 resection, percent viable tumor, total number of lymph nodes resected, positive lymph nodes resected

Induction chemotherapy alone or chemoradiotherapy, transhiatal esophagectomy or thoracotomy 